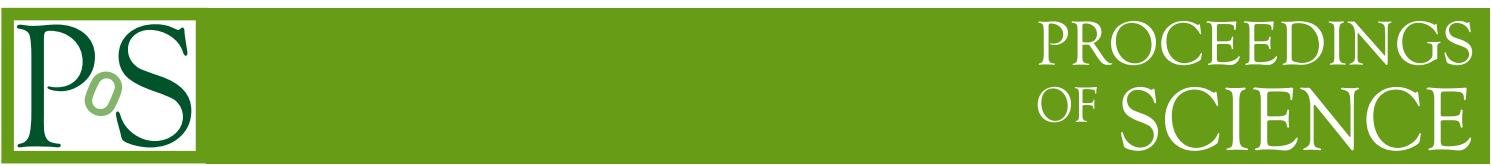

\title{
Constraints of New Physics theories with Gfitter
}

\author{
Max Baak* \\ CERN, CH-1211, Geneva 23, Switzerland \\ E-mail: mbaakecern.ch
}

\begin{abstract}
Results from global fits of several New Physics (NP) models to electroweak precision data are reviewed and discussed. A global fit of the oblique parameters $(S T U)$ to the electroweak data is performed to constrain NP models. The parameter space of the Littlest Higgs Model with $T$-parity is constrained via the oblique parameters. Fit results for the Type-II Two-Higgs-Doublet-Model (2HDM) are presented, using mainly observables from the $B$ and $K$ physics. For $2 \mathrm{HDM}$ we exclude a charged-Higgs mass below $240 \mathrm{GeV}$ at $95 \%$ confidence level. This limit increases towards larger values of $\tan \beta$, where e.g. $M_{H^{ \pm}}<780 \mathrm{GeV}$ is excluded for $\tan \beta=70$.
\end{abstract}

European Physical Society Europhysics Conference on High Energy Physics

July 16-22, 2009

Krakow, Poland

\footnotetext{
*Speaker.

$\dagger$ on behalf of the Gfitter Group (www. cern.ch/Gfitter)
} 


\section{Introduction to Gfitter}

By exploiting contributions from virtual loop corrections, electroweak precision measurements allow us to probe potential effects of new physics, which can itself be at much higher energy scales than the masses of the particles directly involved in experimental reactions. Prominent examples are the electroweak precision measurements, which in the past have been used successfully to predict the top quark mass and (still unmeasured) the Higgs boson mass.

The generic fitting package Gfitter [1] provides a framework for model testing in high-energy physics for the era of the LHC. Gfitter is designed to provide a modular framework for complex fitting tasks and statistical tests, where theoretical models are inserted as plug-in packages. The global electroweak fit of the SM with Gfitter is described in detail elsewhere [1][2].

This paper presents Gfitter results from global fits of several NP models to electroweak precision data. An estimate of the oblique parameters is used to constrain the parameter space of the Littlest Higgs Model (LHM). In addition, a fit of the Type-II Two Higgs Doublets Model (2HDM) is performed using $B$ and $K$ physics observables.

\section{Oblique Parameters}

At low energies, it is commonly assumed that beyond the SM physics appears dominantly through vacuum polarization corrections, also known as oblique corrections. Their effects on electroweak precision observables can be parametrized by three gauge self-energy parameters $(S, T$, $U$ ) introduced by Peskin and Takeuchi [ [⿰]. $S$ describes new physics contributions to neutral current processes at different energy scales. $T$ is sensitive to isospin violation. $U(S+U)$ is sensitive to NP contributions to charged currents. $U$ only sensitive to $W$ mass and width, and is usually very small in NP models (often: $U=0$ ).

Constraints on the $S T U$ parameters are derived from the global fit to the electroweak precision data, presented in [1]. The $S T U$ parameters replace $M_{H}$ and $m_{t}$ as free parameters in the fit. The following results have been obtained from a fit with the radiative corrections from top quark and Higgs boson fixed, assuming $m_{t}=173.1 \mathrm{GeV}$, and $M_{H}=116 \mathrm{GeV}$ (in parentheses $M_{H}=350 \mathrm{GeV}$ ):

$$
\begin{aligned}
S & =0.02(-0.06) \pm 0.11 \\
T & =0.05(0.15) \pm 0.12 \\
U & =0.07(0.08) \pm 0.12,
\end{aligned}
$$

The $S T U$ parameter-space is usually projected onto a two-dimensional parameter space, assuming $U=0$. Fig. 1 1 shows the $68 \%, 95 \%$, and $99 \% \mathrm{CL}$ allowed contours in the $(S, T)$-plane for three different assumptions of $M_{H}$. The experimental constraints on $S, T, U$ are to be compared with specific NP model predictions, e.g. as done in Section . Since the oblique parameters are found to be small, possible NP models may only affect the electroweak observables weakly.

\section{Littlest Higgs Model with T-Parity}

The fine-tuning problem of the Higgs mass parameter, the so-called hierarchy problem, is one of the driving arguments to consider physics beyond the SM. Little Higgs theories provide an 


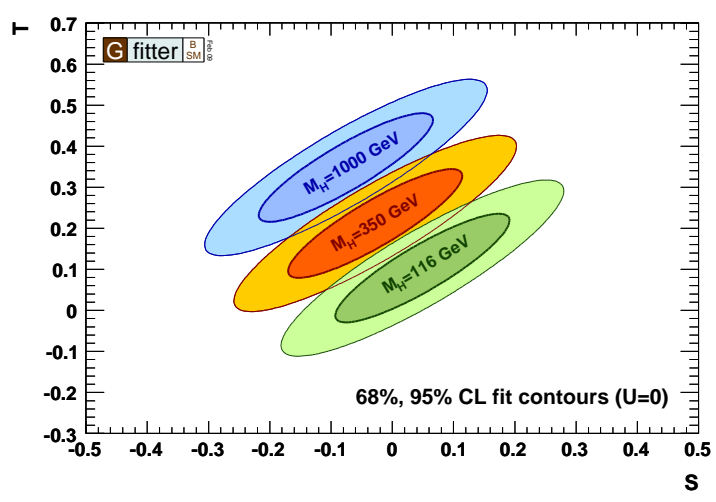

Fig. 1: Allowed contours of $68 \%$ and $95 \% \mathrm{CL}$ in the $(S, T)$-plane obtained from fits with $m_{t}=173.1 \mathrm{GeV}$ and $M_{H}=116,350$, and $1000 \mathrm{GeV}$.

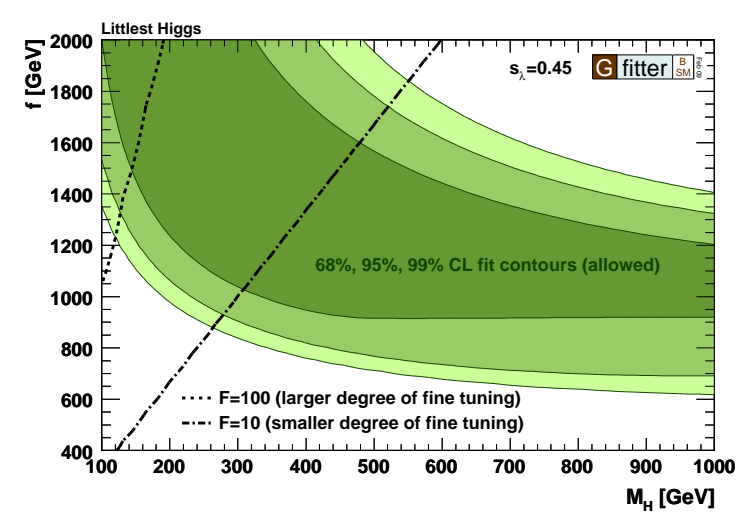

Fig. 2: Allowed contours of $68 \%, 95 \%$, and $99 \% \mathrm{CL}$ obtained from scans of fits with fixed variable pairs $f$ and $M_{H}\left(s_{\lambda}=0.45\right) . \quad F$ is a quantitative measure of fine-tuning (see text).

elegant solution to the hierarchy problem, and are generally based on non-linear $\sigma$ models with a global symmetry broken at an energy scale $f$. The Higgs boson is the lightest of the resulting Goldstone bosons. New SM-like fermions and gauge bosons appear at the TeV scale, in addition to a heavy top-like quark. The Littlest Higgs Model (LHM) [ $\llbracket$ ] is described by SU(5)/SO(5) symmetry breaking, where the conservation of $T$-parity (similar to $R$-parity in SUSY) forbids treelevel contribution from new gauge bosons to the electroweak precision observables.

To constrain the parameter space of the Littlest Higgs Model, the STU parameters of the oblique parameter fit in Section 2 are replaced by their LHM predictions [5]. Including the usual top-quark and Higgs boson masses, $m_{t}$ and $M_{H}$, the floating parameters of the fit are: $f$ the global symmetry breaking scale, $s_{\lambda} \approx m_{T-} / m_{T+}$ in leading order the ratio of masses of the $T$-odd and the $T$-even state from the LHM top sector, and $\delta_{c}$ a order one-coefficient, of which the exact value depends on detail of UV physics. The latter parameter is treated as a theory uncertainty in the fit, with $\delta_{c} \in[-5,5]$.

Fig. 2 shows the $68 \%, 95 \%$, and $99 \% \mathrm{CL}$ allowed contours in the $\left(M_{H}, f\right)$-plane for a fixed value of $s_{\lambda}=0.45$. The parameter $F$ is a quantitative measure of fine-tuning, indicated by the dashed lines. The oblique corrections from the Littlest Higgs Model tend to cancel SM contributions from the top quark and Higgs boson [5]. Consequently, with the inclusion of LHM oblique corrections, the electroweak precision data allow for relatively large Higgs masses. In addition, since larger values of $F$ correspond to larger degrees of fine-tuning, large values of $M_{H}$ are preferred for their smaller fine-tuning.

\section{The Two Higgs Doublet Model}

The Type-II Two Higgs Doublet Models (2HDM) describes an extended Higgs sector where one doublet couples to the up-type fermions, while the other one couples only to the down-type fermions. In this analysis we constrain the mass of the charged Higgs, $M_{H^{ \pm}}$, and the ratio of the 


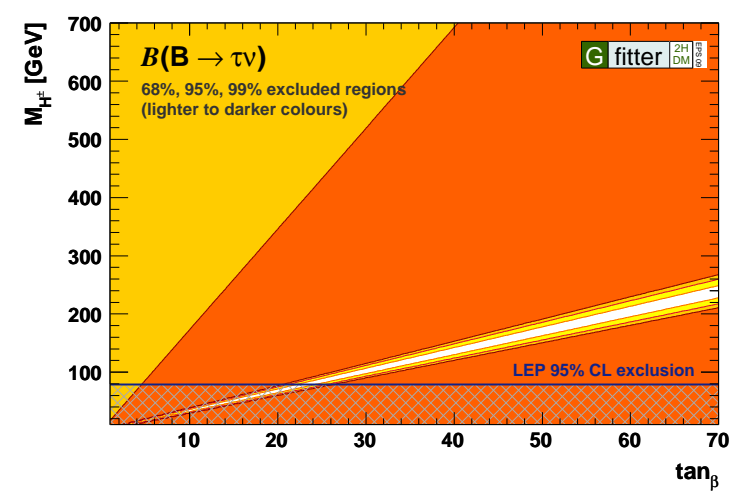

Fig. 3: Constraint from the $B \rightarrow \tau \nu$ branching ratio measurement. The private preliminary average $\mathscr{B}(B \rightarrow \tau v)=(1.73 \pm 0.35) \times 10^{-4}$ of $\mathrm{BaBar}$ and Belle measurements is used as experimental input.

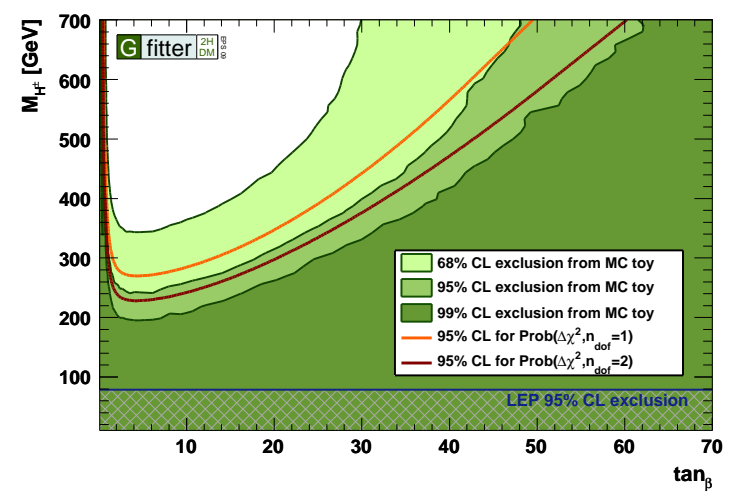

Fig. 4: Exclusion region in the $\left(\tan \beta, M_{H^{ \pm}}\right)$-plane obtained from combined 2HDM constraints. The $95 \%$ $\mathrm{CL}$, as obtained from MC pseudo-experiments, is the middle (wavy) line.

vacuum expectation values of the two Higgs doublets, $\tan \beta$, from indirect measurements. Experimental inputs to the 2HDM fit are observables from the $B$ and $K$ physics sectors, namely: $R_{b}^{0}$, and the branching ratio of $B \rightarrow X_{s} \gamma, B \rightarrow \tau \nu, B \rightarrow \mu \nu, K \rightarrow \mu \nu$, and $B \rightarrow D \tau \nu$. Detailed descriptions of these measurements used and their theoretical 2HDM predictions are found in [i] and references therein.

For each observable, individual constraints have been derived in the $\left(\tan \beta, M_{H^{ \pm}}\right)$plane. The inclusive decay $B \rightarrow X_{s} \gamma$, a flavor-changing neutral current process only occuring at loop-level in the SM, excludes $M_{H^{ \pm}}<220 \mathrm{GeV} / c^{2}$ and $\tan \beta<0.5$ at $95 \% \mathrm{CL}$. With the latest experimental data from $\mathrm{BaBar}$ and Belle, the $B \rightarrow \tau v$ branching ratio gives the single strongest constraint, with a two-fold ambiguity, excluding low Higgs masses at large $\tan \beta$, see Fig. 3 .

For the combined CL calculation in the two-dimensional plane, we have performed Monte Carlo pseudo-experiments in each scan point, which allows to avoid the problem of ambiguities in the effective number of degrees of freedom. The 95\% CL excluded region obtained are indicated in Fig. $⿴$ by the area below the single solid black line. We exclude a charged Higgs mass below $240 \mathrm{GeV}$ independently of $\tan \beta$. This limit increases towards larger $\tan \beta$, e.g. $M_{H^{ \pm}}<780 \mathrm{GeV}$ are excluded for $\tan \beta=70$.

\section{References}

[1] H. Flächer et.al. Eur. Phys. J. C 60 (2009) 543, [arXiv:0811.0009 [hep-ph]].

[2] A. Höcker, "Status of the global electroweak fit of the Standard Model", in these proceedings.

[3] M. E. Peskin and T. Takeuchi, Phys. Rev. D 46, 381 (1992).

[4] N. Arkani-Hamed et al., JHEP 0207, 034 (2002), [arXiv:hep-ph/0206021].

[5] J. Hubisz et al., JHEP 0601135 (2006), [arXiv:hep-ph/0506042]. 